\title{
3D global MHD simulations of a proto-planetary disk: dead zone and large-scale magnetic fields
}

\author{
Natalia Dzyurkevich, Mario Flock and Hubert Klahr \\ Max-Planck Institute for Astronomy, Königstuhl 17, D-69117 Heidelberg, Germany \\ email: natalia@mpia.de
}

\begin{abstract}
We present 3D global MHD simulations of proto-planetary disks calculated with the ZeusMP code. We focus on gas dynamics; the magnetic diffusivity and temperature are fixed during the simulation. A zone with low gas ionization at the midplane is included within \pm 2 scale heights. We mimic the 'snow'-line radius with one order of magnitude jump in magnetic diffusivity $\eta$. Resulting turbulent Maxwell and Reynolds stresses are present at the midplane despite of low ionization. We find no radial inhomogeneities in turbulent $\alpha$ stress for a mild ionization contrast at the 'snow'-line. A smooth azimuthal magnetic field is produced in the dead zone which may be a driving force for a weak accretion flow.
\end{abstract}

Keywords. MHD - turbulence - accretion disks - protoplanetary disks

Magnetic fields play a key role in extracting excess angular momentum in protoplanetary disks, making accretion of the material onto star possible. Radial inhomogeneities in accretion rates, thus building up pressure ridges, have been suggested to be a solution for one of the major problems in the theory of planet formation - building of planetesimals (Kretke \& Lin 2007). In ideally ionized disk, the magneto-rotational instability (MRI) provides velocities high enough to prevent the dust grains from growing. Special events like pressure bumps and absence of radial drift may help to overcome the growth barrier, yet the most severe reason for rapid fragmentation is the turbulent motion itself (Brauer et al. 2008). Therefor we focus on MRI in poorly ionized disk. The properties of global magnetic fields are worth to be investigated in global 3D simulations. The launching of outflows and jets is very sensitive to field topology (Beckwith et al. 2008).

We consider the MRI turbulence in partly-ionized circumstellar disk with a 'dead' zone, including the water sublimation front called 'snow'-line. We solve the set of MHD equations using $3 \mathrm{D}$ global simulations on a spherical grid $(r, \Theta, \phi)$,

$$
\begin{gathered}
\frac{\partial \vec{B}}{\partial t}=\nabla \times[\vec{u} \times \vec{B}-\eta(r, \Theta) \nabla \times \vec{B}], \\
\frac{\partial \vec{u}}{\partial t}=-\nabla P+\rho \nabla \Psi+\frac{1}{4 \pi}[\nabla \times \vec{B}] \times \vec{B}, \\
\frac{\partial \rho}{\partial t}+\nabla \cdot(\rho \vec{u})=0 .
\end{gathered}
$$

The notation is the usual one. $\Psi$ is a point-mass gravitational potential. We use the locally isothermal approach to describe vertically stratified disk, adopting $P=c_{\mathrm{s}}^{2}(r) \rho(r, \Theta)$ with a pressure scale height $H / R=0.07$. We choose a purely azimuthal magnetic 'seed' field, which is force-free and $P_{\text {gas }} / P_{\text {mag }}=25$. The models are listed in Table 1 . Ionization profiles used in model $\mathbf{m} \mathbf{1}$ are based on Turner et al. (2007). The mid-plane values of $\eta$ depend on the presence of ice dust grains, so that $\eta_{a}=0.016$ in the presence of $1 \mu$ 
Table 1. Model properties and midplane $\alpha$-stresses before $\left(\alpha_{\mathrm{b}}\right)$ and behind $\left(\alpha_{\mathrm{a}}\right)$ 'snow'-line.

\begin{tabular}{|c|c|c|c|c|c|c|}
\hline Model & Domain Size & Resolution & $\eta_{\mathrm{b}}$ & $\eta_{\mathrm{a}}$ & $\alpha_{\mathrm{b}}$ & $\alpha_{\mathrm{a}}$ \\
\hline $\mathrm{m} 1$ & {$[8 \mathrm{AU}: 8.4 H: \pi / 4]$} & {$[256: 128: 64]$} & $7.10^{-4}$ & 0.016 & $5.10^{-5}$ & $5.10^{-5}$ \\
\hline $\mathrm{m} 2$ & {$[8 \mathrm{AU}: 8.4 H: \pi / 4]$} & {$[256: 128: 64]$} & 0 & 0.016 & $10^{-3}$ & $10^{-6}$ \\
\hline Mod & Domain Size & Resolution & $\eta_{\mathrm{b}}$ & $\eta_{\mathrm{a}}$ & $\alpha_{\mathrm{b}}$ & $\alpha_{\mathrm{a}}$ \\
\hline $\mathrm{m} 1$ & [8 AU : $8 \triangleright 4 H: \pi \triangleleft 4]$ & {$[256: 128: 64]$} & $7 \triangleright 10^{-4}$ & $0 \triangleright 016$ & $5 \triangleright 10^{-5}$ & $5 \triangleright 10^{-5}$ \\
\hline $\mathrm{m} 2$ & {$[8 \mathrm{AU}: 8 \triangleright 4 H: \pi \triangleleft 4]$} & {$[256: 128: 64]$} & 0 & $0 \triangleright 016$ & $10^{-3}$ & $10^{-6}$ \\
\hline
\end{tabular}
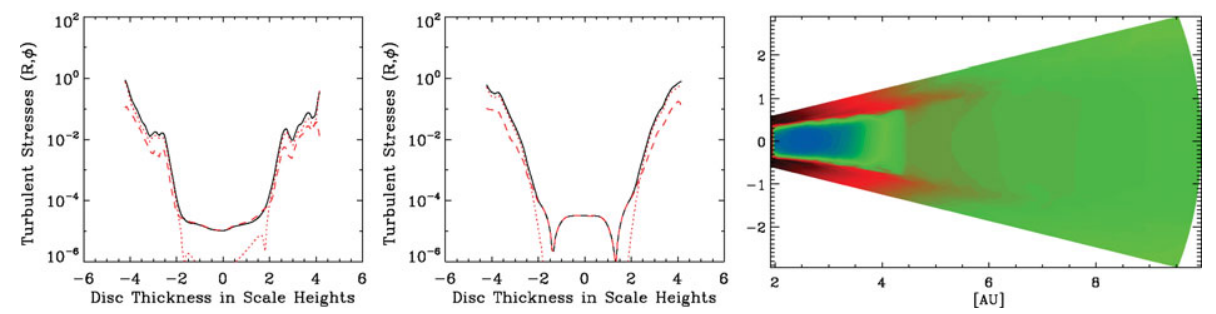

Figure 1. Left: Turbulent stresses between $2 \mathrm{AU}$ and $4 \mathrm{AU}$. Middle: Turbulent stresses between 6AU and 8AU. Red dotted line shows Maxwell stress, red dashed line is Reynolds stress, black line is total stress. Right: azimuthal magnetic field after $t=240$ orbits, red-green-blue colors the change of sign from positive to negative.

ice particles, and $\eta_{b}=7 \cdot 10^{-4}$ if the temperature is high enough for water sublimation. 'Snow'-line is chosen to be at 4.5 AU. Model $\mathbf{m} 2$ is made for less realistic situation when gas is totally ionized before 'snow'-line (Table 1 ).

In case of one order of magnitude jump in ionization (model $\mathbf{m 1}$ ), the total alphastress is around $5 \cdot 10^{-5}$ at the mid-plane, when calculated separately for locations before and after the 'snow'-line (Fig. 1). Model $\mathbf{m 2}$ with extreme high ionization jump (from ideally ionized matter into resistive medium with $1 \mu$-size dust) shows the expected steep jump in stress $\alpha$ at the 'snow'-line (Table 1). This leads to the question which ionization thresholds exist in the real accretion disks. Taking into account the recombination on grains larger then $1 \mu$ may lead to more realistic radial $\alpha$ profile, filling the gap between the models $\mathbf{m} \mathbf{1}$ and $\mathbf{m} \mathbf{2}$. It is note-worthy that the accretion in partly ionized midplane is determined by Reynolds stresses, whereas the magnetic pressure is indeed significantly reduced. The turbulent velocity dispersion is about 0.01 (in local sound speed units) in mid-plane layer, and 0.2 in upper layers. At $4 \mathrm{AU}$, this corresponds to the velocities of about $10 \mathrm{~m} / \mathrm{s}$ and $200 \mathrm{~m} / \mathrm{s}$, making the dust growth through coagulation possible.

In the 'dead'-zone $( \pm 2 H)$ a smooth azimuthal magnetic field $B_{\phi}$ is produced (Fig. 1), confirming the result of Turner \& Sano (2008). For perfectly ionized disk we observe the periodical change of sign in $B_{\phi}$ with respect to the midplane. When a 'dead'-zone is included, only weak periodical change of sign in radial velocity remains. The field symmetry and effects of the boundary conditions, as well as extension of models $\mathbf{m} \mathbf{1}$ and $\mathbf{m} 2$, will be discussed in the following publication.

\section{References}

Kretke, K. A. \& Lin, D. N. C. 2007, ApJ 664, L55

Brauer, F., Henning, Th., \& Dullemond, C. P. 2008, A\& A 487, L1

Beckwith, K., Hawley, J. F., \& Krolik, J. H. 2008, ApJ 678, 1180

Turner, N. J., Sano, T., \& Dziourkevitch, N. 2007, ApJ 659, 729

Turner, N. J. \& Sano, T. 2008, ApJ 679, 131 